\title{
Tidal disruption events from the first XMM-Newton slew survey
}

\author{
P. Esquej ${ }^{1, a}$, R.D. Saxton ${ }^{2}$, S. Komossa ${ }^{3}$ and A.M. Read ${ }^{4}$ \\ ${ }^{1}$ Centro de Astrobiología (INTA-CSIC), ESAC Campus, PO Box 78, \\ 28691 Villanueva de la Cañada, Spain \\ ${ }^{2}$ XMM SOC, ESAC, Apartado 78, 28691 Villanueva de la Cañada, Madrid, Spain \\ ${ }^{3}$ Max-Planck-Institut für Radioastronomie, Auf dem Hügel 69, 53121 Bonn, Germany \\ ${ }^{4}$ Dept. of Physics and Astronomy, Leicester University, Leicester LE1 7RH, UK
}

\begin{abstract}
Observations over the past decade have revealed that supermassive black holes (SMBHs) likely reside at the centres of most or all bulge galaxies. Confirmation of their dormant presence in non-active galaxies is difficult to obtain. An unavoidable consequence of the existence of remnant SMBHs is the detection of a tidal disruption event. This is discovered as flaring radiation produced when a star is tidally disrupted and subsequently accreted by the black hole. Two of these exceptional events have been discovered by XMM-Newton in the first slew catalogue, NGC 3599 and SDSS J132341.97+482701.3. Here we show their evolution up to four years after the peak of the outburst including a detailed analysis of NGC 3599, for which novel follow-up observations are presented here.
\end{abstract}

\section{INTRODUCTION}

Accretion onto supermassive black holes (SMBHs) is believed to play an important role in the formation and evolution of galaxies, where black hole growth and feedback have to be considered in a fully self-consistent manner (e.g. [1]). It has been demonstrated that black holes do exist at the centre of active galaxies, but to detect them lurking in a dormant state is still a challenge. In addition to the stellar dynamics approach, only applicable to very nearby galaxies, the detection of tidal disruption events can be used to unveil the presence of SMBHs in non active galaxies [2]. This phenomenon is observed as flaring radiation produced by the disruption of a star when crossing the tidal radius of the black hole. After one postdisruption orbit, a fraction of the remaining debris bound to the system will be accreted, producing an observable flare of EUV/X-ray radiation decaying as $t^{-5 / 3}$ lasting from months up to several years [3].

Early ROSAT detections of a few tidal disruption events allowed [4] to derive that these objects can be identified for showing extreme variability (factors by up to even thousands in X-rays), extreme $\mathrm{X}$-ray softness in outburst characterized by a black body emission with $k T=0.04--0.1 \mathrm{keV}$, and X-ray peak luminosity up to $\sim 10^{45} \mathrm{erg} \mathrm{s}^{-1}$ that declines at a $t^{-5 / 3}$ rate.

Strong candidates have been identified mainly in X-rays (e.g. [5-11]) and in the UV regime [12-14]. Further attempts have been done in the optical and radio $[15,16]$. The presence of extremely strong and transient coronal lines has also been interpreted as signatures of disruption events $[17,18]$. Although previously detected candidates support theoretical predictions, the detection, confirmation and follow-up of each new case will shed new light on the phenomena due to the paucity of candidates. These occurrences are very rare in the sky. Theoretical models postulate that a tidal disruption event should occur once every $10^{3}-10^{6}$ yr per galaxy [19].

a e-mail: pilar.esquej@cab.inta-csic.es
The XMM-Newton slew source catalogue (XMMSL1; [20]) represents a powerful resource for detecting tidal disruption candidates through comparison with previous $\mathrm{X}$-ray data. Here we present follow-up X-ray observations of two candidates discovered in the XMMSL1 $[9,21]$. We summarise previous data and present new observations for NGC 3599. We are now following a near-real-time approach to the analysis of slew observations to obtain high quality spectral observations around the peak of the outburst. With this program a new candidate has recently been found and presented in [22].

Throughout this paper we will assume a $\Lambda \mathrm{CDM}$ cosmology with $\left(\Omega_{\mathrm{M}}, \Omega_{\Lambda}\right)=(0.3,0.7)$ and $H_{0}=70 \mathrm{~km} \mathrm{~s}^{-1}$ $\mathrm{Mpc}^{-1}$. All X-ray fluxes and luminosities quoted here have been corrected for Galactic absorption.

\section{TIDAL DISRUPTION CANDIDATES}

Correlating the XMMSL1 with the ROSAT all-sky survey (RASS; [23]) we found two galaxies with properties in agreement with the tidal disruption model [9]. Both sources showed high variability with respect to RASS upper limits, a very soft X-ray spectrum in the high-state detection (no photons above $2 \mathrm{keV}$ ) and no evident optical activity according to the literature at the time of detection [24]. All these properties favoured the interpretation of the flaring events as tidal disruptions.

NGC 3599 is an early-type galaxy in the local Universe $(z=0.0028)$. The optical pre-outburst spectrum of the source shows narrow emission lines after the subtraction of the stellar component [21]. Using the diagnostic diagrams in [25], the source is located in the transition region for composite $\mathrm{H}$ II/LINER systems [26]. The spectrum in high state could be fitted with a black body model of $k T=95_{-3}^{+4} \mathrm{eV}$, which is consistent with previous candidates although at the upper range of temperatures. We derived a $0.2-2 \mathrm{keV}$ luminosity of $5.5 \times$ $10^{41} \mathrm{erg} \mathrm{s}^{-1}$. Using upper limits from the RASS, we

This is an Open Access article distributed under the terms of the Creative Commons Attribution License 2.0, which permits unrestricted use, distribution, and reproduction in any medium, provided the original work is properly cited. 
Table 1. Summary of the X-ray follow-up observations.

\begin{tabular}{ccc}
\hline \hline & NGC 3599 & \\
\hline Instrument & Date & Exp (ks) \\
\hline XMM-Newton/EPIC & $2006-06-23$ & 5 \\
Swift $/$ XRT & $2006-12-01$ & 5 \\
Chandra/ACIS-S & $2008-01-26$ & 18 \\
XMM-Newton /EPIC & $2008-12-02$ & 35 \\
Swift/XRT & $2010-10-23$ & 3 \\
\hline \hline \multicolumn{3}{c}{ SDSS J132341.97+482701.3 } \\
\hline Instrument & Date & Exp (ks) \\
\hline XMM-Newton/EPIC & $2006-07-15$ & 5 \\
Swift $/$ XRT & $2007-01-18$ & 5 \\
Swift $/$ XRT & $2011-02-04$ & 5 \\
\hline
\end{tabular}

derived a variability factor of 88 [9]. The source also lies in the field of view of a ROSAT PSPC pointed observation although it did not appear in any of the ROSAT catalogues. The analysis of that exposure revealed a faint detection at the position of NGC 3599 with a soft X-ray luminosity of $4 \times 10^{39} \mathrm{erg} \mathrm{s}^{-1}$. Therefore, the source enhanced its luminosity by a factor of 150 when detected by XMMNewton.

SDSS J132341.97+482701.3 (SDSS J1323 hereinafter) is a non-active galaxy at a redshift of $z=0.087$. Its optical spectrum only shows absorption line features from the host galaxy. The X-ray slew detection of the object showed a flux variation in the $0.2-2 \mathrm{keV}$ energy band by a factor of 83 between the slew detection and its RASS upper limit. The slew observation contained too few counts to allow spectral fitting. A soft unabsorbed X-ray luminosity of $4.8 \times 10^{43} \mathrm{erg} \mathrm{s}^{-1}$ from the slew observation was derived using the typical black body model of $70 \mathrm{eV}$ for tidal disruption events near outburst [4].

Long-slit optical observations of NGC 3599 and SDSS J1323 were performed around three years after the slew detections to obtain post-outburst spectral shapes. They confirmed that both sources did not show any evident optical signature of the flaring event.

\section{X-RAY FOLLOW-UP OBSERVATIONS AND DATA ANALYSIS}

\subsection{XMM-Newton observations}

XMM-Newton target of opportunity (ToO) observations of $5 \mathrm{ks}$ were performed roughly two years after the slew exposures. These were already presented in [21]. See Table 1 for details of all X-ray follow-up observations.

A further $40 \mathrm{ks}$ XMM-Newton observation was performed on NGC 3599 to monitor its luminosity and spectral evolution [27]. All EPIC-pn and MOS exposures were taken in Full Frame (FF) mode with the Medium filter. A standard source detection analysis was performed, which allowed us to improve the position of the slew detection. Source photons were extracted from a circular region of $15^{\prime \prime}$ radius centred on the object position. A circular source-free region on the same chip and radius

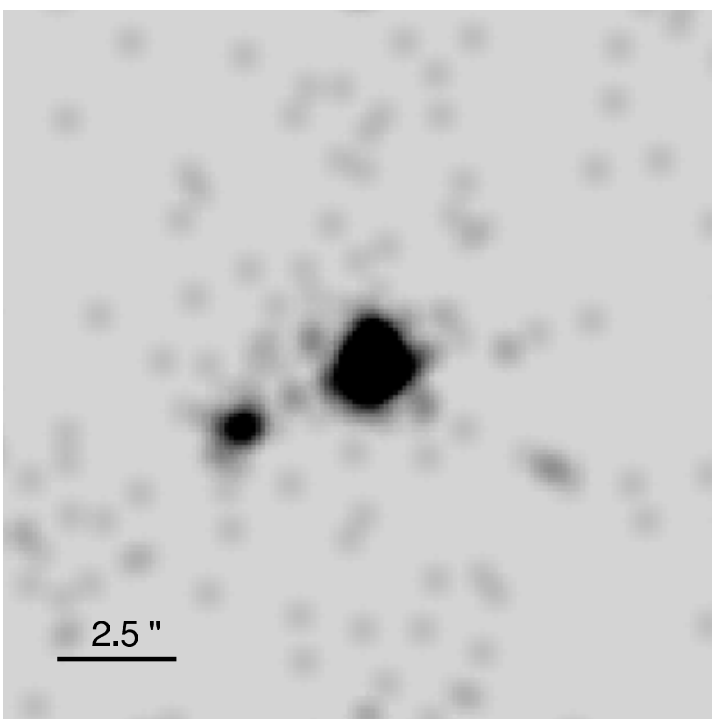

Figure 1. Smoothed Chandra image of $15^{\prime \prime} \times 15^{\prime \prime}$ centred on the nuclear source of NGC 3599. The off-nuclear source can be seen at $2.5^{\prime \prime}$.

of 60 " was used to characterise the background. All EPIC spectra were binned to oversample the instrumental resolution and to have no less than 20 counts in each background-subtracted spectral channel.

\subsection{Swift observations}

Follow-up observations of $5 \mathrm{ks}$ were performed with the X-ray Telescope (XRT) on board the Swift satellite. Both objects turned out to be extremely faint at the time of the Swift observations to apply spectral fits to model the data. To estimate the source fluxes we applied a count rate to flux conversion through PIMMS using the same spectral model derived from the previous XMM-Newton pointed observations. This is the only reasonable assumption that we can make because the behaviour of the post-outburst spectra is a priori unclear. See [21] for a full description.

Further observations by Swift confirmed that there has been no reactivation of NGC 3599 and SDSS J1323 as they were not detected in 2010 and 2011, respectively.

\subsection{Chandra observation}

A 20 ks Chandra observation of NGC 3599 was performed with the Advanced CCD Imaging Spectrometer (ACIS; [28]). Images were corrected for known aspect offsets, reaching a nominal astrometry accuracy of $0.6^{\prime \prime}$ (at the $90 \%$ confidence level).

The Chandra image revealed two X-ray objects separated by $2.5^{\prime \prime}$ within NGC 3599 (about $250 \mathrm{pc}$ at the distance of the source, see Fig. 1). These are a brighter source coincident with the nucleus of the galaxy according to the optical position, and a secondary off-nuclear source. Light curves were inspected, and no variability has been recorded for any of the two sources within the X-ray observation. For the spectral analysis, three extraction regions have been used: two circular regions with radius of 
1.7 and $1.1^{\prime \prime}$ for the bright and faint sources respectively, and a circular source-free region with radius of $5^{\prime \prime}$ to characterise the background. The spectrum of the nuclear source was rebinned to have at least 20 counts in each bin, in order to use the $\chi^{2}$ statistics.

\section{SPECTRAL ANALYSIS}

The spectral analysis of SDSS J1323 was presented in [21]. A simple power-law model with Galactic absorption fits reasonably well the soft X-rays but leaves an apparent high energy excess. Due to the low signal-to-noise ratio of the data we can not improve the fit adding extra model components. This source turned up too faint for posterior follow-up observations with reasonable exposure times.

For NGC 3599 long-term spectral analysis has been performed, thanks to the most recent Chandra and XMMNewton observations. The off-nuclear source detection only has 40 counts, none of them below $0.4 \mathrm{keV}$. Using $\mathrm{C}$-statistics, we derived a best fit using a simple powerlaw model with $\Gamma_{\mathrm{X}}=1.51_{-0.56}^{+0.34}$. Due to the low number of counts one can not make further constrains to the spectral model. The X-ray luminosity in the $0.2-2 \mathrm{keV}$ energy band is $2_{-1}^{+2} \times 10^{38} \mathrm{erg} \mathrm{s}^{-1}$, and $5_{-5}^{+5} \times 10^{38} \mathrm{erg} \mathrm{s}^{-1}$ is the $2-12 \mathrm{keV}$ luminosity. Given the luminosity of the source and the positional offset with respect to the centre of the galaxy, the source can be classified as an ultraluminous X-ray source (ULX) but at the low luminosity end. Due to the very low luminosity and its hard spectrum, one can tentatively propose the possibility of a ULX in a low/hard state.

The similar overall shape of the X-ray spectra derived from the XMM-Newton observations and that of the Chandra nuclear source suggests that the bulk of the X-ray emission detected by XMM-Newton was due to the central object. This is also supported by the low luminosity of the off-nuclear object, which is not likely to contribute much to the combined spectra of the two sources. Data of the XMM-Newton and Chandra pointed observations have been simultaneously fitted using a baseline model plus a constant to allow for different normalisations in the different exposures. We have also added a fixed model to mimic the effect of the faint source in the XMM-Newton observations, this is the power-law model described above. The spectral analysis has been repeated without adding that contribution, and results are analogous.

The best fit is obtained with a power-law plus a black body, the latter to account for the presence of a soft excess expected from thermal emission from an optically-thick accretion disk [29]. The derived parameters are $k T=44_{-17}^{+19} \mathrm{eV}$ and $\Gamma_{X}=2.70_{-0.30}^{+0.33}$. The measured soft excess black body temperature lies within the possible values corresponding to the high energy tail of thermal emission from the hot inner accretion disk [30]. The steep power-law required in the fit differs significantly with that found in standard AGN. The F-test infers a significance of improvement in the fit of $>99 \%$ when adding the black body component to the simple powerlaw. Data and spectral model are presented in Fig. 2.

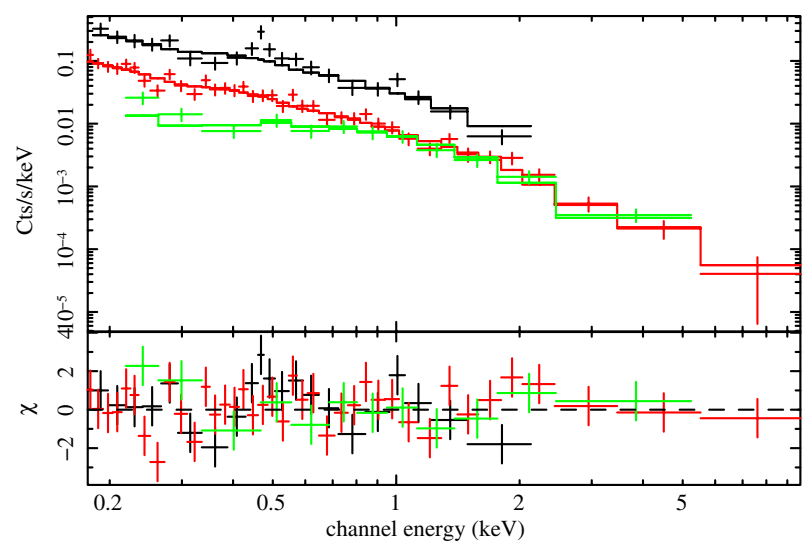

Figure 2. XMM-Newton EPIC-pn ToO (black), Chandra ACIS$\mathrm{S}$ (green) and XMM-Newton (red) X-ray spectra of NGC 3599. Data/model residuals are presented at the bottom.

Models including extra intrinsic absorption do not result in a further improvement of the fit.

\section{X-RAY LIGHT CURVES}

The X-ray flare begins when the most bound material returns to pericentre after completing one orbit. Assuming that the mass distribution of the debris is nearly uniformly distributed as derived from numerical simulations $[3,31]$, the mass rate after one post-disruption orbit should decay as $t^{-5 / 3}$. [32,33] showed that such decreasing law does not hold close to the luminosity maximum and is wavelength dependent. However, it is appropriate for our case as we are dealing with X-ray data at late times after the peak.

Assuming that the debris evolution of our sources is represented by the fallback model of tidal disruption, the derived values for the soft $0.2-2 \mathrm{keV}$ luminosities have been fitted with a $\left(t-t_{\text {disr }}\right)^{-5 / 3}$ decline law, where $t_{d i s r}$ is the disruption time. These fits give

$L_{X}=6.8( \pm 1.2) \times 10^{40}\left[\frac{t-2003.59( \pm 0.06) \mathrm{yr}}{1 \mathrm{yr}}\right]^{-5 / 3} \mathrm{erg} \mathrm{s}^{-1}$

and

$L_{\mathrm{X}}=8.1( \pm 2.9) \times 10^{42}\left[\frac{t-2003.56( \pm 0.10) \mathrm{yr}}{1 \mathrm{yr}}\right]^{-5 / 3} \mathrm{erg} \mathrm{s}^{-1}$

for NGC 3599 and SDSS J1323 respectively. Fig. 3 shows the evolution of the X-ray luminosity and corresponding fitted light curves.

\section{SUMMARY AND CONCLUSIONS}

We have presented X-ray follow-up observations of two tidal disruption candidates detected in the XMMSL1. These were identified as having a very soft spectra in our high state detection and huge variability with respect to the RASS. In addition to summarising previous findings, we have presented new XMM-Newton and Chandra 

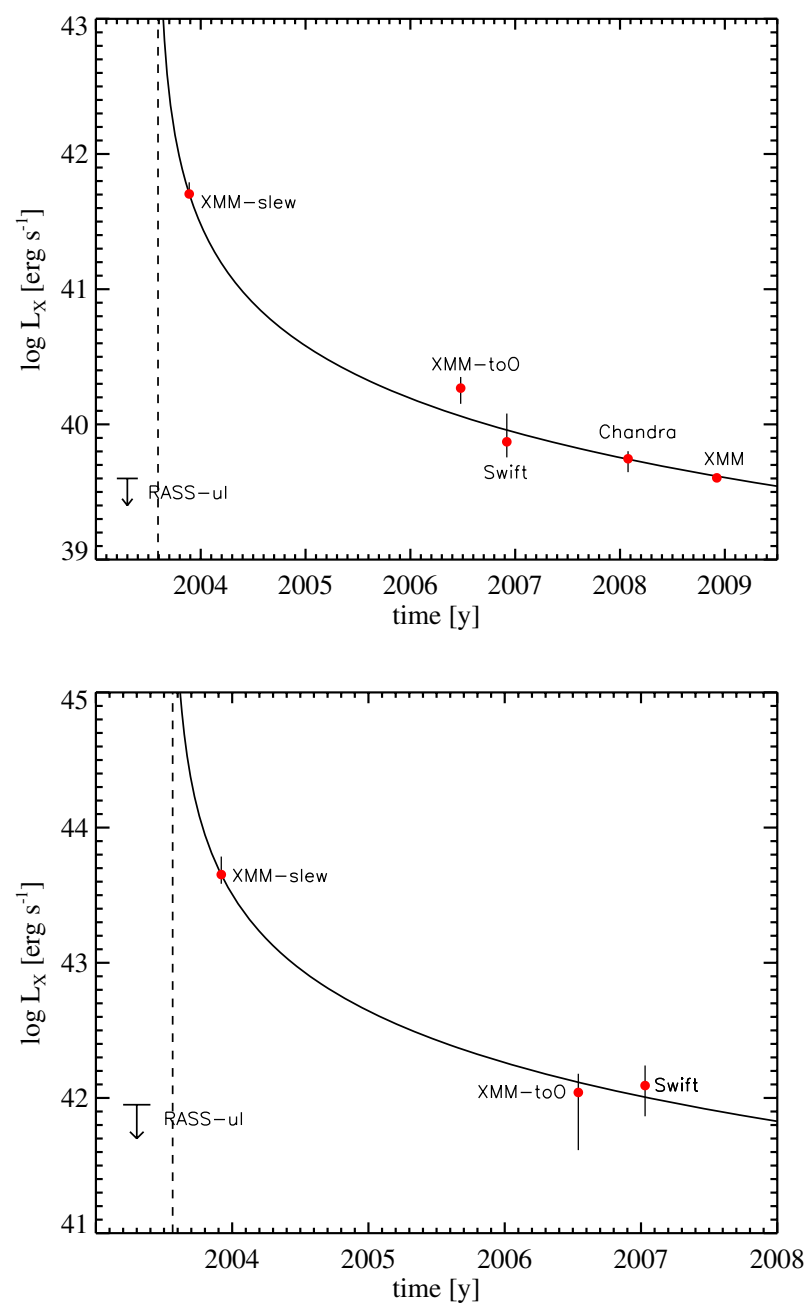

Figure 3. X-ray light curve of NGC 3599 (top) and SDSS J1323 (bottom). RASS upper limit data points have been shifted for plotting purposes. Solid lines are the fitted curves predicted for the fallback stage following a $t^{-5 / 3}$ decline law. Dashed lines show the disruption time.

observations of NGC 3599, which is the nearest tidal disruption event discovered to date. Further details will be presented in [27].

The Chandra observation of NGC 3599 confirmed the presence of nuclear X-ray emission, while also revealed the presence of a secondary off-nuclear source. The source coordinates regarding XMM-Newton coincide with the Chandra position of the bright source within the error circle, which put strong constraints on the X-ray emission detected in previous observations being nuclear. The similarity of general shapes of the X-ray spectra between the XMM-Newton observation and the Chandra nuclear source suggests that the bulk of the X-ray emission, especially at soft energies, was due to the central source. The spectral shape of the source does not seem to have evolved significantly except for a change in intensity. Given that it is not possible to derive a statistically significant fit to the slew spectra, a possible temporal hardening of the source can not be constrained because this is model dependent.

The X-ray luminosity has evolved following the $t^{-5 / 3}$ decaying law, therefore in agreement with the tidal disruption scenario. The detection of these phenomena represents a unique tool to unveil the dormant black hole population, which is fundamental to constrain the accretion history of the Universe.

\section{References}

[1] P.F. Hopkins, L. Hernquist, T.J. Cox, T. Di Matteo, B. Robertson, V. Springel, ApJS 163, 1 (2006)

[2] M. Rees, Nature 333, 523 (1988)

[3] S. Ayal, M. Livio, T. Piran, ApJ 545, 772 (2000)

[4] S. Komossa, Rev. in Modern Astronomy 15, 27 (2002)

[5] N. Bade, S. Komossa, M. Dahlem, A\&A 309, L35 (1996)

[6] S. Komossa, N. Bade, A\&A 343, 775 (1999)

[7] S. Komossa, J. Greiner, A\&A 349, L45 (1999)

[8] D. Grupe, H.C. Thomas, K. Leighly, A\&A 350, L3 (1999)

[9] P. Esquej, R.D. Saxton, M.J. Freyberg, A.M. Read, B. Altieri, M. Sanchez, G. Hasinger, A\&A 462, L49 (2007)

[10] N. Cappelluti, M. Ajello, P. Rebusco, S. Komossa, A. Bongiorno, C. Clemens, M. Salvato, P. Esquej, T. Aldcroft, J. Greiner et al., A\&A 495, L9 (2009)

[11] S.B. Cenko, H.A. Krimm, A. Horesh, A. Rau, D.A. Frail, J.A. Kennea, A.J. Levan, S.T. Holland, N.R. Butler, R.M. Quimby et al., ApJ 753, 77 (2012)

[12] S. Gezari, D.C. Martin, B. Milliard, S. Basa, J.P. Halpern, K. Forster, P.G. Friedman, P. Morrissey, S.G. Neff, D. Schiminovich et al., ApJ 653, L25 (2006)

[13] S. Gezari, S. Basa, D.C. Martin, G. Bazin, K. Forster, B. Milliard, J.P. Halpern, P.G. Friedman, P. Morrissey, S.G. Neff et al., ApJ 676, 944 (2008)

[14] S. Gezari, R. Chornock, A. Rest, M.E. Huber, K. Forster, E. Berger, P.J. Challis, J.D. Neill, D.C. Martin, T. Heckman et al., Nature 485, 217 (2012)

[15] S. van Velzen, G.R. Farrar, S. Gezari, N. Morrell, D. Zaritsky, L. Östman, M. Smith, J. Gelfand, A.J. Drake, ApJ 741, 73 (2011)

[16] D. Giannios, B. Metzger, MNRAS 416, 2102 (2011)

[17] S. Komossa, H. Zhou, T. Wang, M. Ajello, J. Ge, J. Greiner, H. Lu, M. Salvato, R. Saxton, H. Shan et al., ApJ 678, L13 (2008)

[18] T.G. Wang, H.Y. Zhou, S. Komossa, H.Y. Wang, W. Yuan, C. Yang, ApJ 749, 115 (2012)

[19] J. Wang, D. Merrit, ApJ 600, 149 (2004)

[20] R. Saxton, A. Read, P. Esquej, M.J. Freyberg, B. Altieri, D. Bermejo, A\&A 480, 611 (2008)

[21] P. Esquej, R. Saxton, S. Komossa, A. Read, M. Freyberg, G. Hasinger, D. García-Hernández, H. Lu, J.R. Zaurín, M. Sánchez et al., A\&A 489, 543 (2008)

[22] R.D. Saxton, A.M. Read, P. Esquej, S. Komossa, S. Dougherty, P. Rodriguez-Pascual, D. Barrado, A\&A 541, A106 (2012)

[23] W. Voges, B. Aschenbach, T. Boller, H. Bräuninger, U. Briel, W. Burkert, K. Dennerl, J. Englhauser, 
R. Gruber, F. Haberl et al., A\&A 349, 389 (1999)

[24] T.R. Lauer, S.M. Faber, D. Richstone, K. Gebhardt, S. Tremaine, M. Postman, A. Dressler, M.C. Aller, A.V. Filippenko, R. Green et al., ApJ 662, 808 (2007)

[25] G. Kauffmann, T.M. Heckman, C. Tremonti, J. Brinchmann, S. Charlot, S. White, S. Ridgway, J. Brinkmann, et al., MNRAS 346, 1055 (2003)

[26] L.C. Ho, ARA\&A 46, 475 (2008)
[27] P. Esquej, et al., in prep. (2013)

[28] G. Garmire, M. Bautz, P. Ford, J. Nousek, G. Ricker (2003), Vol. 4851 of Proc. SPIE , pp. 28-44

[29] M. Montesinos Armijo, J.A. de Freitas Pacheco, ApJ 736, 126 (2011)

[30] N.I. Shakura, R.A. Syunyaev, A\&A 24, 337 (1973)

[31] C. Evans, C. Kochanek, ApJ 346, L13 (1989)

[32] G. Lodato, A.R. King, J.E. Pringle, MNRAS 392, 332 (2009)

[33] G. Lodato, E.M. Rossi, MNRAS 410, 359 (2011) 